\title{
SMA and ALMA studies of protoplanetary disk formation around low-mass protostars
}

\author{
Shigehisa Takakuwa ${ }^{1}$, Nagayoshi Ohashi ${ }^{1,2}$, Hsi-Wei Yen ${ }^{1}$, \\ Ti-Lin Chou ${ }^{1}$, Kazuya Saigo ${ }^{3}$, Masao Saito ${ }^{4}$, Yusuke $\mathrm{Aso}^{2,5}$, \\ Yuri Aikawa ${ }^{6}$, Shin Koyamatsu ${ }^{2,5}$, Masahiro N. Machida ${ }^{7}$, \\ Sanemichi Z. Takahashi ${ }^{8}$, Kengo Tomida ${ }^{9}$ and Kohji Tomisaka ${ }^{10}$ \\ ${ }^{1}$ Academia Sinica Institute of Astronomy and Astrophysics, PO Box 23-141, Taipei 10617, \\ Taiwan, email: takakuwa@asiaa.sinica.edu.tw \\ ${ }^{2}$ Subaru Telescope, National Astronomical Observatory of Japan, 650 North A'ohoku Place, \\ Hilo, HI 96720, USA \\ ${ }^{3}$ Department of Physical Science, Graduate School of Science, Osaka Prefecture University, \\ 1-1 Gakuen-cho, Naka-ku, Sakai, Osaka 599-8531, Japan \\ ${ }^{4}$ Nobeyama Radio Observatory, National Astronomical Observatory of Japan, Minamimaki, \\ Minamisaku, Nagano 384-1805, Japan \\ ${ }^{5}$ Department of Astronomy, Graduate School of Science, The University of Tokyo, 7-3-1 \\ Hongo, Bunkyo-ku, Tokyo 113-0033, Japan \\ ${ }^{6}$ Center for Computational Sciences, University of Tsukuba, 1-1-1 Tennoudai, \\ Tsukuba 305-8577, Japan \\ ${ }^{7}$ Department of Earth and Planetary Sciences, Faculty of Sciences, Kyushu University, \\ Fukuoka 812-8581, Japan \\ ${ }^{8}$ Department of Physics, Kyoto University, Oiwake-cho, Kitashirakawa, Sakyo-ku, \\ Kyoto 606-8502, Japan \\ ${ }^{9}$ Department of Earth and Space Science, Osaka University, 1-1 Machikaneyama, \\ Toyonaka, Osaka 560-0043, Japan \\ ${ }^{10}$ National Astronomical Observatory of Japan, Osawa 2-21-1, Mitaka, Tokyo 181-8588, Japan
}

\begin{abstract}
We report our systematic survey observations of protostellar sources with the SubMillimeter Array (SMA) and Atacama Large Millimeter/submillimeter Array (ALMA). The purpose of our survey is to investigate formation mechanism of protoplanetary disks, precursors of planetary systems, out of $\sim 1000 \mathrm{AU}$-scale protostellar envelopes surrounding the protostars. We found that in the early protostars (B335, NGC1333 IRAS 4B), the envelopes do not show significant rotating motions but infalling motions toward the central protostars. In more evolved protostars (L1527 IRS, L1448-mm, L1551 IRS 5), the envelopes are infalling and rotating with the conserved specific angular momenta (that is, $v_{r o t} \propto r^{-1}$ ). In most evolved sources (L1551 NE, TMC-1A, L1489 IRS) large-scale ( $\gtrsim 100$ AU) disks in Keplerian rotation or protoplanetary disks are evident. These results demonstrate a systematic evolutionary trend of envelope gas motions toward the disk formation.
\end{abstract}

Keywords. Protostellar Envelopes, Keplerian Disks, SMA, ALMA

\section{Introduction}

Keplerian disks are ubiquitous around T-Tauri stars, and are considered to be precursors of planetary systems, "protoplanetary disks". Indeed, recent high-resolution millimeter and submillimeter continuum observations of the disk around HL Tau with ALMA have unveiled presence of seven ringlike gaps in the disk, evidence for presence of planets (ALMA Partnership et al. 2015). The formation mechanism of such protoplanetary disks should define the initial conditions of subsequent disk evolution toward planet formation. 


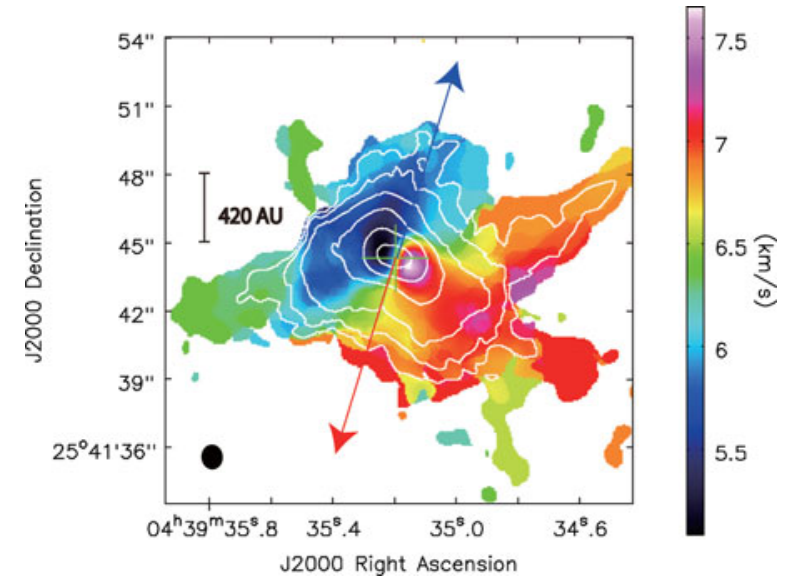

Figure 1. Moment 0 (white contours) and 1 (color) maps of the $\mathrm{C}^{18} \mathrm{O}(J=2-1)$ emission in TMC-1A observed with ALMA, taken from Aso et al. (2015). A cross shows the position of the central protostar (continuum emission peak), and blue and red arrows the direction of the blueshifted and redshifted molecular outflows observed in the ${ }^{12} \mathrm{CO}(J=2-1)$ emission. A filled ellipse at the bottom-left corner denotes the ALMA synthesized beam $\left(1^{\prime \prime} 06 \times 0\right.$ '. 90 ; P.A. $\left.=-176^{\circ}\right) .($ A color version of this figure is available online.)

Our research team has initiated a large collaborative study of formation mechanism of protoplanetary disks from protostellar envelopes surrounding the protostars with the SMA and ALMA. We have observed eight protostellar sources ranging from early Class 0 to late Class I stages with ALMA and/or SMA, and investigated the gas motions in the envelopes. Below, we will show examples of our results.

\section{TMC-1A}

TMC-1A is a late Class I protostar $\left(T_{b o l}=172 \mathrm{~K}\right)$ located in the Taurus region. Fig. 1 shows moment 0 (white contours) and 1 (color) maps of the $\mathrm{C}^{18} \mathrm{O}(J=2-1)$ emission in TMC-1A observed with ALMA (Aso et al. 2015). There appears a $\sim 1000$-AU scale $\mathrm{C}^{18} \mathrm{O}$ feature elongated approximately perpendicular to the associated molecular outflows (blue and red arrows). This $\mathrm{C}^{18} \mathrm{O}(J=2-1)$ emission most likely traces the molecular envelope component associated with the protostar. The eastern and western parts of the emission component are blueshifted and redshifted, respectively, and the most blueshifted and redshifted velocities appear close to the protostar.

Fig. 2 compares distributions of the high- and low-velocity blueshifted and redshifted $\mathrm{C}^{18} \mathrm{O}$ emission in TMC-1A. The high-velocity blueshifted and redshifted $\mathrm{C}^{18} \mathrm{O}$ emission are located to the east and west of the protostar, respectively. The direction of the velocity gradient is almost perpendicular to the direction of the associated molecular outflow, and there is no clear emission overlap of the blueshifted and redshifted emission. On the other hand, the low-velocity blueshifted and redshifted emission exhibit significant spatial overlaps, and the direction of the overall velocity gradient is tilted with respect to that of the high-velocity emission.

From our protostellar envelope + disk modeling, we found that the high-velocity components can be explained as an $r \sim 100$-AU scale Keplerian disk orbiting a protostar with a dynamical mass of $0.68 M_{\odot}$, while the low-velocity components an infalling and rotating protostellar envelope. The rotational profile of the envelope follows $v_{\text {rot }} \propto r^{-1}$, that is, rotation with the conserved specific angular momentum, and the infalling velocity is $\sim 3$ times smaller than free-fall velocity yielded by the dynamical mass of the protostar. 

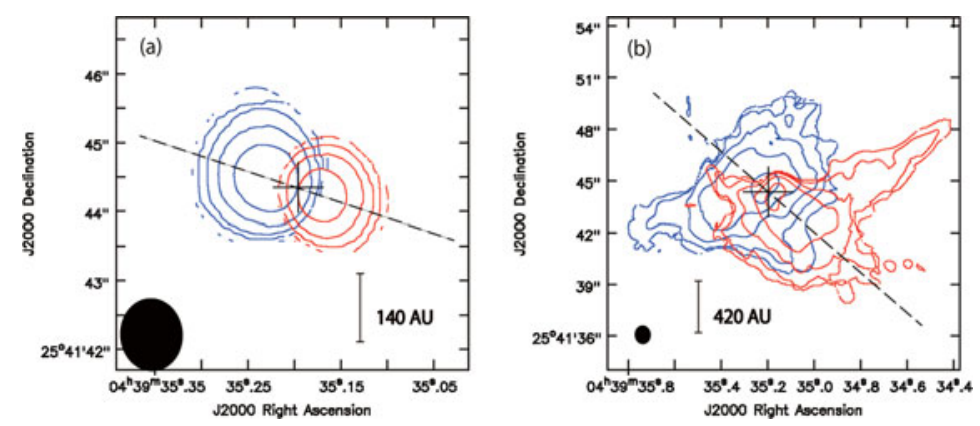

Figure 2. ALMA maps of the blueshifted (blue contours) and redshifted $\mathrm{C}^{18} \mathrm{O}(J=2-1)$ emission (red) at (a) high-velocity $\left(|\Delta V|>2.0 \mathrm{~km} \mathrm{~s}^{-1}\right)$ and (b) low-velocity $(|\Delta V|<2.0 \mathrm{~km}$ $\mathrm{s}^{-1}$ ) ranges in TMC-1A, taken from Aso et al. (2015). The dashed lines show the directions of the observed velocity gradients. (A color version of this figure is available online.)
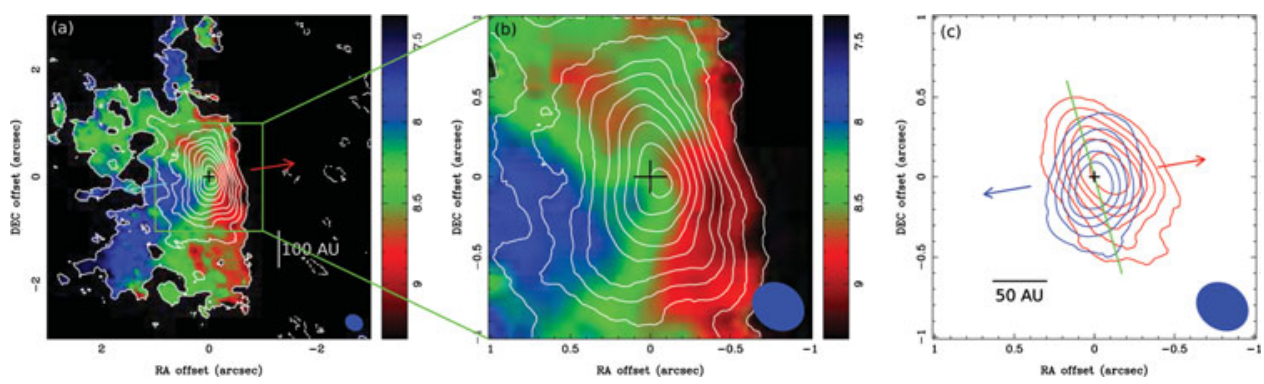

Figure 3. (a) ALMA moment 0 map (contours) overlaid on the moment 1 map (color scale, units in $\left.\mathrm{km} \mathrm{s}^{-1}\right)$ of the $\mathrm{C}^{18} \mathrm{O}(J=2-1)$ emission in B335. A cross indicates the protostellar position, and blue and red arrows denote the directions of the associated blueshifted and redshifted outflows, respectively. (b) Zoom-up view of the central $2^{\prime \prime} \times 2^{\prime \prime}$ region delineated by the green box in panel (a). (c) Moment 0 map of the high-velocity $\left(|V|>1.2 \mathrm{~km} \mathrm{~s}^{-1}\right)$ blueshifted and redshifted $\mathrm{C}^{18} \mathrm{O}$ emission in $\mathrm{B} 335$. The green line shows the direction of the major axis of the $1.3 \mathrm{~mm}$ continuum emission. These figures are from Yen et al. (2015b). (A color version of this figure is available online.)

These results indicate that the protoplanetary disk is being formed out of the infalling envelope, where the infalling velocity is decelerated as the material approaches close to the disk.

\section{B335}

Whereas our ALMA observations of a late-phase protostar TMC-1A found a welldeveloped Keplerian disk embedded in the rotating and infalling protostellar envelope, there are other protostellar sources without any detectable disk or even envelope rotation. Fig. 3 shows results of our ALMA observations of the Class 0 protostar B335 in the $\mathrm{C}^{18} \mathrm{O}$ (2-1) emission (Yen et al. 2015b). Fig. 3 (a) shows the presence of a $\sim 500$-AU scale protostellar envelope elongated perpendicular to the associated molecular outflow. While the protostellar envelope exhibits a conspicuous velocity gradient along the minor axis originated from the infalling motion, there is no clear velocity gradient along the major axis (i.e., rotation) down to the radial scale of $\lesssim 50 \mathrm{AU}$.

The absence of the envelope rotation implies that the outermost radius of the Keplerian disk is as small as $\lesssim 1-3 \mathrm{AU}$. These results indicate that in the early Class 0 protostar of B335 the envelope material is actively infalling toward the protostar and the disk 
Table 1. Evolution of Envelope Gas Motion toward Protoplanetary-Disk Formation

\begin{tabular}{|lcclll}
\hline Protostars & Class & $T_{b o l}[\mathbf{K}]$ & Envelope & $R_{k e p}[\mathbf{A U}]^{1}$ & Our Publ. $^{2}$ \\
\hline B335 & 0 & 31 & infall+slow rotation & $<1-3$ & $\mathrm{a}, \mathrm{b}, \mathrm{c}, \mathrm{d}, \mathrm{e}$ \\
NGC1333 IRAS $4 \mathrm{~B}$ & 0 & 54 & infall+slow rotation & $<5$ & $\mathrm{c}, \mathrm{d}$ \\
L1527 IRS & $0-\mathrm{I}$ & 59 & slow infall $+r^{-1}$ rotation & 54 & $\mathrm{c}, \mathrm{d}, \mathrm{f}$ \\
L1448-mm & O-I & 69 & infall $+r^{-1}$ rotation & $\sim 150$ & $\mathrm{c}, \mathrm{d}$ \\
L1551 NE & I & 91 & slow infall+slow rotation & 300 & $\mathrm{~g}, \mathrm{~h}, \mathrm{i}$ \\
L1551 IRS 5 & I & 92 & slow infall $+r^{-1}$ rotation & 64 & $\mathrm{j}, \mathrm{k}$ \\
TMC-1A & I & 172 & slow infall $+r^{-1}$ rotation & 100 & $\mathrm{c}, \mathrm{l}$ \\
L1489 IRS & I-II & 238 & infall $+r^{-1}$ rotation & $>300$ & $\mathrm{c}, \mathrm{m}$ \\
\hline
\end{tabular}

Notes:

${ }^{1}$ Estimated outermost radius of the protoplanetary disk in Keplerian rotation.

${ }^{2}$ (a) Yen et al. 2010; (b) Yen et al. 2011; (c) Yen et al. 2013; (d) Yen et al. 2015a; (e) Yen et al. 2015b;

(f) Ohashi et al. 2014; (g) Takakuwa et al. 2012; (h) Takakuwa et al. 2013; (i) Takakuwa et al. 2014;

(j) Takakuwa et al. 2004; (k) Chou et al. 2014; (1) Aso et al. 2015; (m) Yen et al. 2014.

formation around the protostar has not been well proceeded, in contrast with the latephase Class I protostar TMC-1A.

\section{Summary}

So far, we have completed ALMA and/or SMA observations and detailed modeling of eight protostellar sources. Table 1 summarizes the observed protostars, their evolutional stages, measured envelope motions and outermost radii of the Keplerian disks, and our publications. Here, "slow rotation" denotes no detectable rotational motion at $r \lesssim 500$ $\mathrm{AU}$, and "slow infall" the infalling velocity $\gtrsim 3$ times smaller than the free-fall velocity. In the early Class 0 protostars of B335 and NGC1333 IRAS 4B, the envelopes do not show clear rotating motions but infalling motions toward the central protostars. In L1527 IRS, L1448-mm, and L1551 IRS 5, the envelopes are infalling and rotating with the conserved specific angular momenta (that is, $v_{\text {rot }} \propto r^{-1}$ ), while the radii of the inner Keplerian disks are still small $(r \lesssim 100 \mathrm{AU})$. In the most evolved sources of L1551 NE, TMC$1 \mathrm{~A}$ and L1489 IRS, large-scale disks in Keplerian rotation or protoplanetary disks are present. These results demonstrate the systematic evolutionary trend of the envelope motion toward the disk formation. In the early-stage protostars the envelopes have just started collapsing and only a small amount of the envelope angular momentum is carried in, and thus the disk formation is not yet initiated. As more envelope materials fall in, more angular momenta are carried toward the center, initiating the disk formation. In the most evolved protostellar sources disk formation has been well developed.

\section{References}

ALMA Partnership, Brogan, C. L., Pérez, L. M., Hunter, T. R., et al. 2015, ApJ, 808, 3 Aso, Y., Ohashi, N., Saigo, K., et al. 2015, ApJ, 812, 27 Chou, T.-L., Takakuwa, S., Yen, H.-W., Ohashi, N., \& Ho, P. T. P. 2014, ApJ, 796, 70

Ohashi, N., Saigo, K., Aso, Y., et al. 2014, ApJ, 796, 131

Takakuwa, S., Ohashi, N., Ho, P. T. P., et al. 2004, ApJ (Letters), 616, L15

Takakuwa, S., Saito, M., Lim, J., et al. 2012, ApJ, 754, 52

Takakuwa, S., Saito, M., Lim, J., \& Saigo, K. 2013, ApJ, 776, 51

Takakuwa, S., Saito, M., Saigo, K., et al. 2014, ApJ, 796, 1

Yen, H.-W., Takakuwa, S., \& Ohashi, N. 2010, ApJ, 710, 1786

Yen, H.-W., Takakuwa, S., \& Ohashi, N. 2011, ApJ, 742, 57

Yen, H.-W., Takakuwa, S., Ohashi, N., \& Ho, P. T. P. 2013, ApJ, 772, 22

Yen, H.-W., Takakuwa, S., Ohashi, N., et al. 2014, ApJ, 793, 1

Yen, H.-W., Koch, P. M., Takakuwa, S., et al. 2015a, ApJ, 799, 193

Yen, H.-W., Takakuwa, S., Koch, P. M., et al. 2015b, ApJ, 812, 129 